https://doi.org/10.23913/rics.v8i16.79

Artículos Científicos

\title{
Estado de la prescripción por enfermería en la región Costa de Oaxaca
}

\author{
Current State of Prescription of Medications by Licensed Medical Nurses in the \\ Costa de Oaxaca Region
}

\section{Estatuto de prescrição por enfermagem na região da Costa de Oaxaca}

\author{
Josué Vásquez Martínez \\ Universidad de la Costa, Oaxaca, México \\ josue_vm@uncos.edu.mx \\ https://orcid.org/0000-0003-0883-2576
}

\section{Resumen}

La prescripción enfermera ha crecido significativamente en todo el mundo durante la última década. Recientemente en México se adicionó el artículo 28 Bis a la Ley General de Salud: ahí se enuncia que los licenciados en enfermería podrán prescribir cuando no se cuente con los servicios de un médico. A la fecha no existe ningún estudio a nivel nacional y mucho menos uno a nivel local que muestre el estado que guarda la prescripción por enfermería. Consecuentemente, el objetivo de este trabajo se centra en describir el estado de esta práctica en la región Costa de Oaxaca. Para ello se empleó un estudio de tipo cuantitativo, con un análisis descriptivo y de alcance transversal. La población estuvo constituida por 86 licenciados en enfermería adscritos a la Jurisdicción Sanitaria 04 Costa del estado de Oaxaca y que laboran en el primer nivel de atención. Y la muestra final constó de 11 licenciados en enfermería seleccionados por conveniencia. Se diseñó una encuesta con 10 preguntas y para el análisis de los datos se empleó el paquete estadístico SPSS versión 22, en donde se utilizaron las funciones de estadísticos descriptivos y respuestas múltiples.

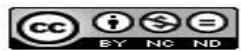




\section{Revista Iberoamericana de las Ciencias de la Salud}

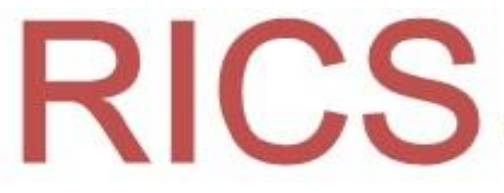

De entre los resultados, $54.5 \%$ de los participantes dijo contar con los conocimientos adecuados para realizar el diagnóstico correcto de la patología. Sin embargo, un idéntico porcentaje mencionó no haber escuchado nunca sobre el artículo 28 Bis de la Ley General de Salud, hasta que fue mencionado en la aplicación de la encuesta. Pese a ello, el total de los participantes prescribe en los centros de salud. El medicamento más prescrito fue el paracetamol (12.4\%). Por último, el total de los participantes estuvo de acuerdo en que el profesional de enfermería debe prescribir. A partir de lo anterior en este estudio se plantea que los licenciados en enfermería que laboran en el primer nivel de atención en centros de salud de la Secretaría de Salud de la región Costa del estado de Oaxaca sí prescriben y asumen dicha práctica como una función del gremio.

Palabras clave: investigación en enfermería, prescripción enfermera, primer nivel de atención.

\section{Abstract}

The prescription of medications by licensed medical nurses has grown significantly worldwide during the last decade. Mexico recently added Article 28 Bis to the General Health Law, which states that licensed medical nurses may prescribe when they do not have the services of a doctor. To date there is no study at the national level and much less one at a local level which shows/demonstrates the state of prescriptions by nurses. Therefore, the objective of this work focuses on describe the current state of this practice in the Costa de Oaxaca region.

A quantitative study was used, with a descriptive analysis and cross-sectional scope. The population was constituted by 86 licensed nurses assigned to the Sanitary Jurisdiction 04 Coast of the state of Oaxaca and who work in the first level of attention. The sample consisted of 11 licensed medical nurses selected for convenience. A survey with 10 questions was designed and for the analysis of the data the statistical package SPSS version 22 was used, where the functions of descriptive statistics and multiple answers are used.

As part of the results, $54.5 \%$ of the participants said they had the adequate knowledge to make the correct diagnosis of the pathology. Likewise, a $54.5 \%$ of the participants mentioned that they had never heard about Article 28 Bis of General Health Law, until it was mentioned in the application of the survey. $100 \%$ of the participants prescribe medication in the health centers. The

\section{(c) $\mathrm{BP}$ (⿻)}

Esta obra está bajo licencia internacional Creative commons Reconocimiento-Nocomercial- 
most prescribed medication was paracetamol (12.4\%). And 100\% of the participants mentioned that the nursing professional should prescribe.

Based on the results obtained in this study, it is proposed that licensed nurses who work in the first level of care in health centers of the Secretary of Health of the Costa region of the state of Oaxaca, prescribe medication and assume this practice as a function of the profession.

Keywords: nursing research, nurse prescribing, primary level of healthcare.

\section{Resumo}

A prescrição de enfermagem tem crescido significativamente em todo o mundo durante a última década. Recentemente, no México, o artigo 28 Bis foi acrescentado à Lei Geral de Saúde: afirma que os graduados em enfermagem poderão prescrever quando não tiverem os serviços de um médico. Até o momento não há estudo em nível nacional e muito menos um em nível local que mostre o estado que mantém a prescrição pela enfermagem. Consequentemente, o objetivo deste trabalho é descrever o estado dessa prática na região da Costa de Oaxaca. Para tanto, foi utilizado um estudo quantitativo, com uma análise descritiva e um escopo transversal. A população foi constituída por 86 egressos em enfermagem lotados na Jurisdição Sanitária 04 Litoral do estado de Oaxaca e que atuam no primeiro nível de atenção. E a amostra final consistiu de 11 graduados em enfermagem selecionados por conveniência. Uma pesquisa com 10 questões foi elaborada e para a análise dos dados foi utilizado o pacote estatístico SPSS versão 22, onde foram utilizadas as funções de estatística descritiva e respostas múltiplas.

Entre os resultados, 54,5\% dos participantes disseram ter o conhecimento correto para fazer o diagnóstico correto da patologia. No entanto, uma porcentagem idêntica mencionou nunca ter ouvido falar sobre o artigo 28 bis da Lei Geral de Saúde, até que foi mencionado na aplicação da pesquisa. Apesar disso, o número total de participantes prescreve nos centros de saúde. O medicamento mais prescrito foi o paracetamol (12,4\%). Por fim, o total de participantes concordou que o profissional de enfermagem deve prescrever. Pelo exposto, neste estudo, afirma-se que os graduandos de enfermagem que atuam no primeiro nível de atenção em centros de saúde do Ministério da Saúde da região da Costa do estado de Oaxaca prescrevem e assumem essa prática em função do guilda

\section{(c) $\mathrm{BP}$ (⿻)}


Palavras-chave: pesquisa em enfermagem, prescrição de enfermagem, primeiro nível de atenção.

Fecha Recepción: Noviembre 2018

Fecha Aceptación: Mayo 2019

\section{Introducción}

La prescripción enfermera es la capacidad del enfermero o enfermera, en el marco de la dirección, evaluación y prestación de los cuidados de enfermería, de "seleccionar, guiados por el criterio profesional, diferentes materiales, productos, dispositivos y medicación encaminados a satisfacer las necesidades de salud del usuario y la población, apoyados por el juicio clínico enfermero y administrados en forma de cuidados" (Consejo General de Enfermería, s. f.). Bulechek y McCloskey (1992) citado en Dandicourt, Martínez y Velásquez (2011) la definen de forma más general como cualquier cuidado directo que el enfermero realiza en beneficio de la persona; ese cuidado directo incluye tratamientos iniciados por los enfermeros, tratamientos iniciados en función del diagnóstico médico y actividades diarias esenciales para la persona.

La prescripción de enfermería ha crecido significativamente en todo el mundo durante la última década, debido a los esfuerzos de diferentes actores que se han visto reflejados en el desarrollo de reformas sustanciales de leyes y de políticas que han fomentado y apoyado que las enfermeras asuman papeles de prescripción tanto en ámbitos de atención como en la salud comunitaria. En consecuencia, el papel de la enfermería en la administración de medicamentos actualmente atraviesa por una gran transformación (Bellaguarda, Nelson, Padilha y Caravaca, 2015).

Dicha práctica acata los dictados de la normatividad vigente de cada país o incluso de cada estado o provincia. Según el Royal College of Nursing (2014), en el Reino Unido la prescripción enfermera ha crecido significativamente durante la última década gracias a las más de 54000 enfermeras y matronas prescriptoras, quienes, conforme al National Health System, prescribieron 12.8 millones de medicamentos y productos sanitarios de enero a marzo de 2010. Además, en 2006, las enfermeras prescriptoras tuvieron pleno acceso a la British National Formulary, lo que las ha puesto a la par con los médicos respecto a la capacidad prescriptiva. Para calificar como prescriptor,

\section{(C) $\rightarrow$ FO}

Esta obra está bajo licencia

internacional Creative commons

Reconocimiento-Nacomercial-

SinObrasDerivadas 4.0 . 


\section{Revista Iberoamericana de las Ciencias de la Salud}

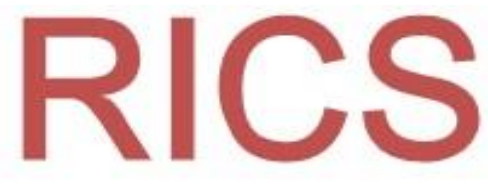

las enfermeras deben acreditar un curso reconocido por el Consejo de Enfermería y Obstetricia (NMC) en una de las universidades del Reino Unido. La evidencia en dicho territorio demuestra que la prescripción enfermera mejora el cuidado del paciente, garantiza el acceso oportuno a los medicamentos y tratamientos y aumenta la eficiencia de los servicios médicos, lo que redunda, esto último, en un mayor tiempo para la atención de pacientes con más complejas necesidades de cuidado de la salud. Todo esto se ve respaldado por los pacientes, quienes reportan un alto nivel de satisfacción y confianza en la prescripción enfermera.

En Suecia la prescripción surge en 1988 como una alternativa para garantizar la atención sanitaria y el acceso a fármacos en zonas geográficas muy alejadas y de difícil acceso, en el entorno de un servicio nacional de salud donde la densidad de profesionales de enfermería es elevada. En 1997 el Gobierno sueco evaluó las prácticas prescriptivas de las enfermeras distritales. Al obtener resultados positivos, se decidió extender la autoridad de prescripción a las enfermeras que trabajan con las autoridades locales en la atención de los ancianos. En este país, las matronas utilizan tanto modelos independientes como colaborativos al ejercer esta práctica, la mayoría preferentemente en la planificación de la familia con la prescripción de los anticonceptivos orales (Fernández, 2016; Dandicourt et al., 2011).

En España se ha iniciado una carrera de fondo para derogar el Real Decreto 954/2015, el cual limita la participación de los enfermeros y las enfermeras en la prestación farmacéutica del sistema sanitario y que ha tenido un rechazo profesional casi unánime. Se necesita, por tanto, modificar la Ley del Medicamento mediante la publicación de un real decreto que permita el desarrollo de la prescripción enfermera en cada uno de los sistemas sanitarios que componen el Sistema Nacional de Salud de España, ya que sin ello las enfermeras españolas no podrán avanzar en este tipo de actuaciones dentro de la prestación farmacéutica en sus correspondientes servicios de salud. Sin embargo, existe una excepción en el ámbito estatal español: el caso de la comunidad autónoma y el Servicio Sanitario Público de Andalucía (SSPA), que publicó un decreto gracias al cual las enfermeras de Andalucía pueden: 1) usar e indicar medicamentos que no necesitan receta médica y prescribir productos sanitarios y 2) realizar prescripción colaborativa (intervención sobre dosis, frecuencia y suspensión del fármaco) para el seguimiento individualizado de tratamientos farmacológicos vinculados a procesos asistenciales. Entre algunos resultados de interés obtenidos por los profesionales de enfermería en el SSPA, destacan los siguientes: 7600 profesionales (c) 9 (9) 


\section{Revista Iberoamericana de las Ciencias de la Salud}

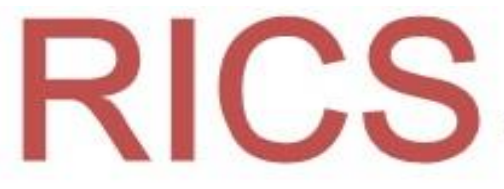

enfermeros que realizan prescripción independiente; $61 \%$ de los productos sanitarios han sido prescritos por enfermeras (en algunas áreas y distritos sanitarios de atención primaria se ha llegado hasta $80 \%$ ); en relación con la prescripción colaborativa, se han publicado cuatro protocolos de seguimiento farmacológico enfermero para personas con enfermedades crónicas (riesgo vascular, anticoagulación oral, sedación paliativa y diabetes) en los que se han acreditado hasta el momento 173 enfermeras y otras 570 se encuentran en proceso de acreditación (Rodríguez, de la Fuente, Casado y Ayuso, 2015).

En Canadá las enfermeras generalistas, es decir, enfermeras licenciadas con más preparación académica y experiencia, que tienen y demuestran las competencias necesarias para actuar de forma autónoma en el diagnóstico, requisición e interpretación de exámenes diagnósticos, son aquellas que legalmente pueden prescribir medicamentos y realizar procedimientos específicos dentro de su ámbito de práctica. Tan solo por citar un caso, en la provincia de Ontario esta actividad se realiza en todas las áreas de la práctica enfermera. En los hospitales, un profesional de enfermería tiene — de acuerdo con la distribución de los pacientes o mecanismos de regulación internos de las instituciones de salud - un grupo de pacientes bajo su responsabilidad, por lo que todo el proceso de investigación diagnóstica y tratamiento se define por este profesional. Los límites interprofesionales en el sistema de salud se basan en la comunicación interprofesional y de colaboración. Así, el ejercicio de la enfermería se reconoce como una acción complementaria de otro profesional de la salud y no como apoyo práctico. El proyecto de ley número 179/2009 extiende la autonomía de las enfermeras generalistas en cuanto a la prescripción de medicamentos, autoridad legal adicional, y admisión y alta de pacientes dentro de áreas específicas de la atención de salud y conocimiento profesional (Bellaguarda et al., 2015).

En Estados Unidos de América, las enfermeras prescriben como parte de una práctica avanzada desde 1997. Aunque el nivel de autoridad prescriptora depende de la legislación de cada estado, en general se plantea que deben estar registradas a nivel nacional (Dandicourt et al., 2011).

En Cuba, por su parte, en el año 2008 se aprobó y puso en vigor en todos los niveles del sistema nacional de salud un documento denominado Regulación de la Práctica de Enfermería, el cual reflejó el inicio de la prescripción enfermera. Este documento declara entre algunas de las funciones asistenciales del licenciado y especialista en enfermería la toma de decisión, control y ejecución al administrar analgésico y antipiréticos; decisión y ejecución en la colocación y

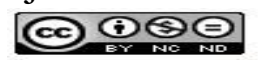




\section{Revista Iberoamericana de las Ciencias de la Salud}

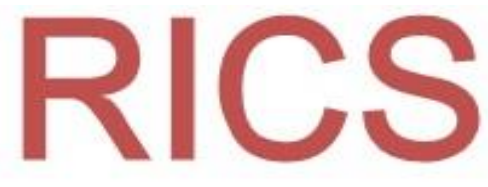

permanencia de la sonda nasogástrica ante la presencia de vómitos; decisión y ejecución en la colocación y permanencia de la sonda vesical en colaboración con el médico según el caso; utilización de soluciones y otros elementos durante las curas de heridas, desinfecciones concurrentes y terminales (Dandicourt et al., 2011).

En Brasil la práctica de la prescripción enfermera inició en 1990 y ha tenido una expansión real y de aplicabilidad desde el año 2006. Dicha práctica sigue protocolos que especifican los fármacos que las enfermeras pueden prescribir. Como parte de la atención primaria de la salud, las enfermeras realizan consultas de enfermería previamente programadas en todo el sistema de salud y atienden áreas específicas, tales como el área de la salud de la mujer, la salud del adulto y anciano, la salud de niños y adolescentes y de grupos relacionados con enfermedades crónicas específicas, a saber, la diabetes, hipertensión y otras. Los clientes de servicios de salud en primer lugar reciben la consulta de enfermería y luego se derivan a un médico cuando es necesario (Bellaguarda et al., 2015).

En el caso de México, el 7 de febrero de 2012, la Cámara de Diputados aprobó con 256 votos a favor, 15 en contra y 6 abstenciones el dictamen que faculta a los y las licenciadas en enfermería para prescribir recetas médicas a los pacientes, con lo que se garantiza que los pacientes tengan acceso a sus medicamentos a través de una prescripción médica elaborada por un profesional de dicho ámbito (Pacheco, 2012). El pleno avaló la adición del artículo 28 Bis a la Ley General de Salud y su publicación en el Diario Oficial de la Federación (DOF). En este artículo se enuncia que los "Licenciados en Enfermería (...) únicamente podrán prescribir cuando no se cuente con los servicios de un médico, aquellos medicamentos del cuadro básico que determine la Secretaría de Salud" (DOF, 2012).

Aunado a lo anterior, recientemente en el DOF se ha publicado el procedimiento y los criterios a los que deberán sujetarse los licenciados en enfermería y pasantes en servicio social para poder prescribir medicamentos. En dicho acuerdo se enuncia que deberán utilizar el formato de receta institucional que para el efecto proporcione la institución en la que presten sus servicios. Igualmente, se precisa que en aquellos casos en los que no se encuentre un médico, homeópata o cirujano dentista en el establecimiento de atención primaria a la salud que pueda realizar la valoración, diagnóstico y prescripción correspondiente, los licenciados en enfermería podrán

\section{(C) $\mathrm{BSO}$}

Esta obra está bajo licencia internacional Creative commons

Reconocimiento-Nacomercial-

SinObrasDerivadas 4.0 . 


\section{Revista Iberoamericana de las Ciencias de la Salud}

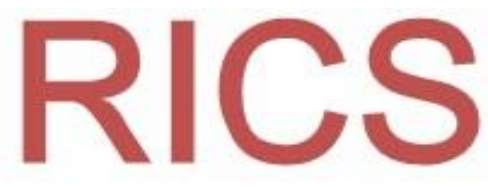

prescribir un total de 93 medicamentos correspondientes al Cuadro Básico de Insumos del Sector Salud (DOF, 2017).

Para satisfacer la gran necesidad en la materia, se consolidó la Red Mexicana de Enfermería en la Prescripción de Medicamentos, integrada por asociaciones, sociedades, colegios, federaciones, escuelas, universidades, el Instituto Politécnico Nacional (IPN), el Instituto Mexicano del Seguro Social (IMSS), el Instituto de Seguridad y Servicios Sociales de los Trabajadores del Estado (Issste) y un numeroso grupo de profesionales de enfermería independientes (Pérez, 2013). Cabe señalar que esta asociación llevó a cabo la primera reunión nacional respecto a la reforma líneas arriba mencionada: "Evidencias de Cuidado de la Facultad para la Prescripción de Medicamentos por Profesionales de Enfermería", con sede en la Universidad Autónoma de Chihuahua, con el objetivo de lograr un análisis profundo y una capacitación de profesionales de esta área en México (Núñez, 2014).

Actualmente, la Comisión Permanente de Enfermería trabaja en una iniciativa en la que se precisen los atributos y responsabilidades que debe de cumplir el profesional de enfermería, en apego a las disposiciones jurídicas, normativas, así como los fundamentos de farmacología clínica requeridos para ejecutar una prescripción de medicamentos segura (Pérez, 2013).

En nuestro país, el profesional de enfermería prescribe de forma independiente en el tratamiento de lesiones cutáneas (heridas, quemaduras o úlceras por presión), prescribe vacunas, micronutrientes, anticonceptivos, vida suero oral, antiinflamatorios no esteroideos, productos para la incontinencia urinaria, etc. Se trata de casos en los que se siguen protocolos previamente establecidos por un equipo multidisciplinario para la toma de decisiones fundamentadas en la permanencia y constante contacto con los pacientes (Cerezo, Espinoza, Techalotzi, y Tlalpan, 2016; Pérez, 2013).

Cuando se habla de prescripción, esta tiende a ser enmarcada en la capacidad de los médicos y no en la de otros profesionales de la salud; se comete, por tanto, una reducción de este ejercicio, así como de la extensión de recetas. Esta idea se mantiene presente aún en nuestros tiempos. Sirva como ejemplo lo siguiente: al elaborar y diseñar una propuesta de receta enfermera, Cerezo et al. (2016) señalaron que para un grupo de profesionales en enfermería con grado universitario resultó confusa la conceptualización del término receta enfermera, y no estuvieron de acuerdo con lo discutido aquí, ya que, en su opinión, la receta forma parte del trabajo del médico y no de la (a) (9) 


\section{Revista Iberoamericana de las Ciencias de la Salud}

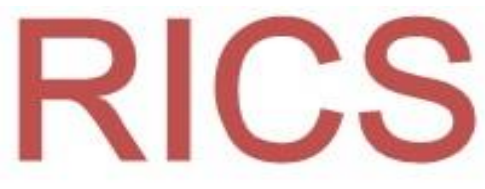

enfermera; las prescripciones, recalcaron, solo las escribe el médico, y que el área de enfermería se encarga únicamente de los cuidados y solo por indicación médica.

No obstante, la prescripción enfermera tiene cabida en las actividades de la profesión, ya que esta incluye cualquier cuidado directo que los profesionales de enfermería realizan en beneficio de los pacientes. Dicha práctica supone una parte importante en el desarrollo profesional de la enfermería y entronca con los planes de cuidados; es, incluso, una consecuencia lógica de los mismos. En este sentido, puede decirse que la prescripción forma una parte intrínseca del trabajo enfermero que empata con las demandas sociales de la población, un creciente avance de la profesión y, por tanto, cambios en el rol profesional, un mayor perfil y la realización de funciones avanzadas en la práctica (Dandicourt et al., 2011).

Ahora bien, para el desarrollo de la autoridad prescriptiva en enfermería se precisa de un planteamiento integrado que tome en consideración lo siguiente:

1) Los aspectos de reglamentación. Por ejemplo, asegurar una legislación que autorice a los enfermeros prescribir, el tipo de formación para tener autoridad prescriptiva, los fármacos o drogas que se podrán prescribir; en fin, se necesitan herramientas legales propias y autónomas de la disciplina que logren integrar el manejo farmacológico en beneficio del paciente.

2) Las competencias para desarrollar la función. Es necesario que el profesional de la enfermería esté calificado para recetar determinados fármacos y para prescribir cuidados en pro de la salud; tiene que saber incorporar por medio del criterio profesional diferentes productos sanitarios, accesorios y medicamentos encaminados a satisfacer la necesidad de salud del paciente; necesita de capacidades que le permitan combinar funciones con el resto del equipo de salud.

3) La formación necesaria para dotar a las enfermeras de las competencias deseadas. El cuidado profesional significa diagnosticar y tratar los problemas de salud, los cuales requieren de conocimientos, actitudes y destrezas que solamente se adquieren con estudios sistemáticos y ordenados. El profesional de enfermería tiene la obligación de actualizarse día con día en los conocimientos y habilidades técnicas de su especialidad, con objeto de ofrecer a su paciente la mejor atención posible, mediante el estudio continuo del avance de los fármacos, la literatura médica-científica, la asistencia a cursos especializados, a congresos y otras reuniones profesionales, sesiones académicas o asociaciones colegiadas (Dandicourt et al., 2011; Cerezo et al., 2016).

\section{(c) क्षेत्}

Esta obra está bajo licencia internacional Creative commons

Reconocimiento-NaComercial- 


\section{Revista Iberoamericana de las Ciencias de la Salud}

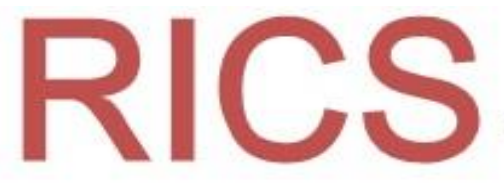

La investigación al respecto es escasa. Se han descrito las labores de prescripción de medicamentos por enfermería (Céspedes, Bedoya y Palacios, 2010), el desempeño de los enfermeros en la prescripción de anticonceptivos hormonales (Dombrowski, Pontes y Assis, 2013), los beneficios de la prescripción enfermera (Vidal, Torres y Carmena, 2016), etc... Sin embargo, a pesar de que la prescripción por enfermería fue aprobada en el año 2012 en México, hasta el momento no se ha encontrado material bibliográfico que muestre los resultados de la aplicabilidad de dicha ley o que exponga los factores que determinan esta práctica. A la fecha no existe ningún estudio a nivel nacional, y mucho menos uno a nivel local, que muestre el estado que guarda la prescripción por enfermería.

Por otro lado, los tópicos de la prescripción enfermera son un reto para el gremio; deben ser atendidos oportunamente por los organismos (asociaciones, colegios y sociedades) para vigilar la praxis que, sin lugar a duda, impactará en el desarrollo de la enfermería en México (Pérez, 2013). Lo que demuestra la necesidad de generar un marco de referencia al respecto para mejorar esta práctica profesional. Por tanto, el presente estudio tiene como objetivo describir el estado de la prescripción por enfermería en la región Costa de Oaxaca.

\section{Materiales y métodos}

El presente estudio fue de tipo cuantitativo, con un análisis descriptivo y de alcance transversal. La población estuvo constituida por 86 licenciados en enfermería adscritos a la Jurisdicción Sanitaria 04 Costa del estado de Oaxaca y que laboran en el primer nivel de atención; en la población no se consideró a quienes laboran en Centros de Salud con Servicios Ampliados (CESSA) y centros de salud urbanos. La muestra final para este estudio constó de 11 licenciados en enfermería seleccionados por conveniencia.

Se diseñó una encuesta con 10 preguntas agrupadas en tres secciones que se crearon tomando en cuenta a Dandicourt et al. (2011), quienes mencionan que para el desarrollo de la autoridad prescriptiva en enfermería se precisa de un planteamiento integrado que tome en consideración los siguientes puntos: 1) los aspectos de reglamentación, 2) las competencias para desarrollar la función y 3) la formación necesaria para dotar a las enfermeras de las competencias deseadas. Además, se incluyó un grupo extra (circunstancias y frecuencia con la que se prescribe)

\section{(C) GES}

Esta obra está bajo licencia

internacional Creative commons

Reconocimiento-Nacomercial-

SinObrasDerivadas 4.0 . 
para complementar esta información, ya que la práctica clínica es un componente fundamental en el proceso de enseñanza y aprendizaje de enfermería; es lo que se llama conocimiento en acción. Dicho conocimiento le permitirá al profesional de enfermería resolver situaciones que se le planteen en un futuro (Tesa, 2014).

La presente investigación se llevó de acuerdo con lo establecido en la Ley General de Salud en Materia de Investigación para la Salud, y se empleó un consentimiento informado que el participante firmó al aceptar participar.

Para el análisis de los datos se empleó el paquete estadístico SPSS versión 22, en donde se utilizaron las funciones de estadísticos descriptivos y respuestas múltiples.

\section{Resultados}

Las encuestas fueron recabadas del 12 de abril al 25 de mayo del 2017 en 10 centros de salud pertenecientes a la Jurisdicción Sanitaria 04 Costa. Participaron 11 licenciados en enfermería: 10 mujeres y 1 varón. En la figura 1 se puede apreciar un mapa de la región Costa del estado de Oaxaca; allí, por medio de puntos, se muestran las unidades médicas en las que laboran las y los licenciados en enfermería que participaron en esta investigación. Como se puede observar, las unidades médicas de cinco participantes se encuentran en el distrito de Jamiltepec, dos en el distrito de Juquila y cuatro en el distrito de Pochutla. El participante con mayor edad tenía 48 años y el menor 22 años, la edad promedio fue de 35.09 años. El total de los participantes ratificó sus estudios como Lic. en Enfermería: 54.5 \% realizó sus estudios en la Unidad Académica No. 3 de Ometepec Guerrero, perteneciente a la Universidad Autónoma de Guerrero (UAGro); $18.2 \%$ en el Instituto de Ciencias y Estudios Superiores de Tamaulipas; $9.1 \%$ en la Unidad Académica No. 2 de Acapulco Guerrero, perteneciente a la UAGro; $9.1 \%$ en la Universidad de Querétaro, y $9.1 \%$ en el Colegio de Estudios y Especialidades del Estado de Oaxaca.

Figura 1. Centros de Salud donde laboran los participantes

\section{(a) 98}




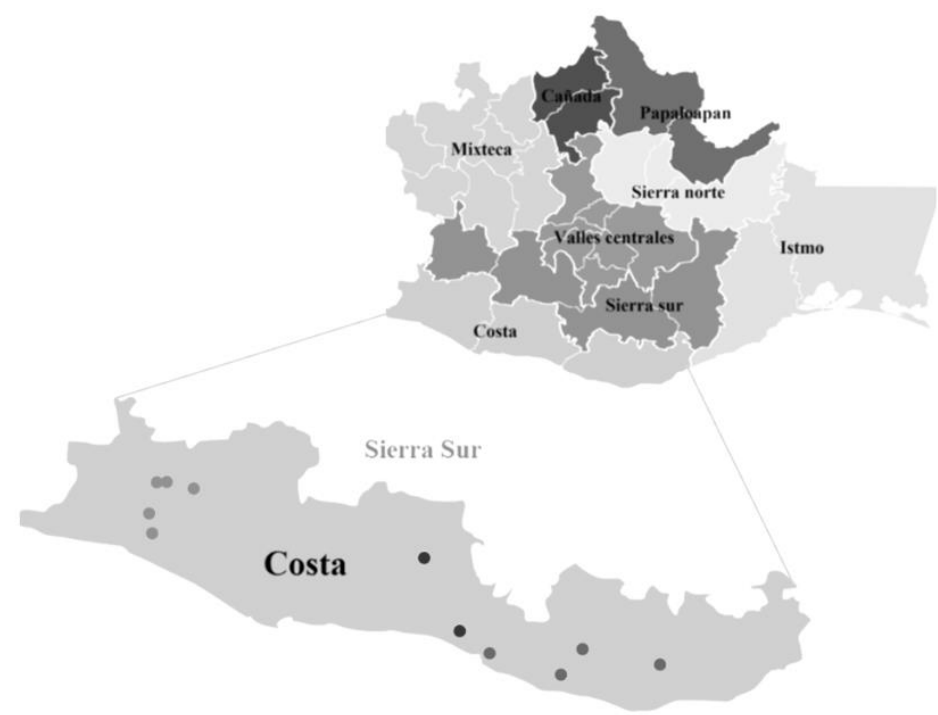

Nota: En el mapa de la región Costa y de izquierda a derecha, primero aparecen los centros de salud pertenecientes al distrito de Jamiltepec, luego aparecen los que se encuentran en Juquila y, por último, los de Pochutla.

Fuente: Elaboración con base en imágenes obtenidas de Google

De acuerdo con la operacionalización de las variables, los resultados son los siguientes.

\section{Niveles de conocimientos indispensables para la prescripción (competencias y formación)}

En el nivel de preparación en cuanto a farmacología, los resultados muestran que las asignaturas relacionadas con la farmacología que cursaron durante la carrera son las siguientes: Farmacología 1 (33.3 \%), Farmacología 2 (22.2 \%), Medicina Alternativa (7.4 \%), Biología 1 (3.7 \%), Biología 2 (3.7\%), Dietoterapia (3.7\%), Nutrición (3.7\%), Fisiología (3.7 \%), Anatomía (3.7 \%), Docencia (3.7 \%), Etimologías (3.7\%), Enfermería Pediátrica (3.7 \%) y Salud en la Comunidad (3.7\%). Por otro lado, $99.9 \%$ de los participantes mencionó no haber recibido algún curso de actualización en farmacología. En cuanto a conocimientos relacionados con el diagnóstico correcto de la patología, $54.5 \%$ de los participantes dijo contar con los conocimientos adecuados, frente a $18.2 \%$ que dijo no contar con los conocimientos adecuados y $27.3 \%$ que dijo no saber si cuenta con los conocimientos adecuados para realizar el diagnóstico correcto de la patología. Con

\section{(c) 9 (i)}


respecto a la comprensión del artículo 28 Bis de la Ley General de Salud, $54.5 \%$ de los participantes mencionó no haber escuchado nunca sobre dicho artículo hasta que fue mencionado en la aplicación de la encuesta.

\section{Circunstancias y frecuencia con la que se prescribe}

En relación con los ámbitos en los que se prescribe, $100 \%$ de los participantes prescribe en los centros de salud con 107 prescripciones al mes, un promedio de 9.73 prescripciones y una desviación estándar de 11.288 (tabla 1).

Tabla 1. Ámbitos de prescripción enfermera

\begin{tabular}{|l|c|c|c|}
\hline $\begin{array}{l}\text { Lugar de } \\
\text { prescripción }\end{array}$ & $\begin{array}{c}\text { Número de } \\
\text { participantes }\end{array}$ & $\begin{array}{c}\text { Porcentaje de } \\
\text { prescripción }\end{array}$ & $\begin{array}{c}\text { Total de } \\
\text { prescripciones al } \\
\text { mes }\end{array}$ \\
\hline Centro de Salud & 11 & $68.8 \%$ & 107 \\
\hline Hogar & 3 & $18.8 \%$ & 7 \\
\hline $\begin{array}{l}\text { Consultorio de } \\
\text { enfermería }\end{array}$ & 1 & $6.3 \%$ & 10 \\
\hline Farmacia & 1 & $6.3 \%$ & 20 \\
\hline Total & & $100 \%$ & 144 \\
\hline
\end{tabular}

Fuente: Elaboración a partir de resultados obtenidos por SPSS versión 22.

Las tres patologías más atendidas son las siguientes: las infecciones respiratorias agudas $(17.8 \%)$, la diabetes $(15.6 \%)$ y las enfermedades diarreicas agudas (13.3\%) (ver tabla 2).

Tabla 2. Patologías en las que se prescribe

\begin{tabular}{|l|l|l|}
\hline Patologías & Número de veces por mes & Porcentaje \\
\hline
\end{tabular}

\section{(c) $\mathrm{BP}$ No}




\section{Revista Iberoamericana de las Ciencias de la Salud}

\begin{tabular}{|l|c|c|}
\hline Infecciones respiratorias agudas & 8 & $17.8 \%$ \\
\hline Diabetes & 7 & $15.6 \%$ \\
\hline Enfermedades diarreicas agudas & 6 & $13.3 \%$ \\
\hline Hipertensión & 4 & $8.9 \%$ \\
\hline Infección de Vías Urinarias & 3 & $6.7 \%$ \\
\hline Fiebre & 3 & $6.7 \%$ \\
\hline Faringoamigdalitis & 1 & $2.2 \%$ \\
\hline Salmonelosis & 1 & $2.2 \%$ \\
\hline Fiebre tifoidea & 1 & $2.2 \%$ \\
\hline Parasitosis & 1 & $2.2 \%$ \\
\hline Infección de garganta & 1 & $2.2 \%$ \\
\hline Planificación familiar & 1 & $2.2 \%$ \\
\hline Gastritis & 1 & $2.2 \%$ \\
\hline Lumbalgia & 1 & $2.2 \%$ \\
\hline Dengue & 1 & $2.2 \%$ \\
\hline Reacciones alérgicas & 15 & $100 \%$ \\
\hline Total & 1 & \\
\hline
\end{tabular}

Fuente: Elaboración propia.

Los tres medicamentos más prescritos son paracetamol (12.4\%), metformina (6.7 \%) y captopril $(6.7 \%)$ (véase tabla 3$)$.

Tabla 3. Fármacos prescritos

\section{(c) $\mathrm{BP}$ (⿻)}


Revista Iberoamericana de las Ciencias de la Salud

\begin{tabular}{|c|c|c|}
\hline Fármaco & $\begin{array}{c}\text { Número de } \\
\text { participantes que lo } \\
\text { prescriben }\end{array}$ & Porcentaje \\
\hline Paracetamol & 11 & $12.4 \%$ \\
\hline Metformina & 6 & $6.7 \%$ \\
\hline Captopril & 6 & $6.7 \%$ \\
\hline Glibenclamida & 5 & $5.6 \%$ \\
\hline Ampicilina & 5 & $5.6 \%$ \\
\hline Clorfenamina & 4 & $4.5 \%$ \\
\hline Amoxicilina con ácido clavulánico & 4 & $4.5 \%$ \\
\hline Loratadina & 3 & $3.4 \%$ \\
\hline Naproxeno & 3 & $3.4 \%$ \\
\hline Diclofenaco & 3 & $3.4 \%$ \\
\hline Trimetoprima con sulfametoxazol & 3 & $3.4 \%$ \\
\hline Ambroxol & 3 & $3.4 \%$ \\
\hline Metronidazol & 2 & $2.2 \%$ \\
\hline Albendazol & 2 & $2.2 \%$ \\
\hline Metoprolol & 2 & $2.2 \%$ \\
\hline Omeprazol & 2 & $2.2 \%$ \\
\hline Cefalexina & 2 & $2.2 \%$ \\
\hline Electrolitos orales & 2 & $2.2 \%$ \\
\hline Ketorolaco & 2 & $2.2 \%$ \\
\hline Enalapril & 2 & $2.2 \%$ \\
\hline Ácido acetilsalicílico & 1 & $1.1 \%$ \\
\hline Propranolol & 1 & $1.1 \%$ \\
\hline Hidróxido de aluminio & 1 & $1.1 \%$ \\
\hline Hidróxido de magnesio & 1 & $1.1 \%$ \\
\hline Ranitidina & 1 & $1.1 \%$ \\
\hline Eritromicina & 1 & $1.1 \%$ \\
\hline
\end{tabular}

\section{(a) 98}




\section{Revista Iberoamericana de las Ciencias de la Salud}

\begin{tabular}{|l|l|l|}
\hline Miconazol & 1 & $1.1 \%$ \\
\hline Isoconazol & 1 & $1.1 \%$ \\
\hline Clioquinol & 1 & $1.1 \%$ \\
\hline Nifedipino & 1 & $1.1 \%$ \\
\hline Lidocaína & 1 & $1.1 \%$ \\
\hline Butilhioscina & 1 & $1.1 \%$ \\
\hline Penicilina & 1 & $1.1 \%$ \\
\hline Ciprofloxacino & 1 & $1.1 \%$ \\
\hline Ácido fólico & 1 & $1.1 \%$ \\
\hline Fumarato ferroso & 1 & $1.1 \%$ \\
\hline Dicloxacilina & 1 & $1.1 \%$ \\
\hline
\end{tabular}

Fuente: Elaboración propia.

\section{Factores legales y operacionales de la prescripción (reglamentación)}

Con respecto a qué opinaban del artículo 28 Bis de la Ley General de Salud, $81.8 \%$ de los participantes mencionó que no debe desaparecer y $18.2 \%$ mencionó que debe de modificarse. En cuanto a las implicaciones bioéticas y legales de la prescripción enfermera, todos los participantes mencionaron que el profesional de enfermería debe prescribir.

\section{Discusiones}

La prescripción por enfermería es un tema novedoso y requiere ser difundido por las autoridades sanitarias, ya que $54.5 \%$ de los participantes mencionó no haber escuchado nunca sobre el artículo 28 Bis de la Ley General de Salud, sino hasta que fue mencionado en la aplicación de la encuesta; se requiere, por tanto, conocer el marco legal para no incurrir en acciones negativas; es decir, actos que deriven en negligencia, imprudencia e impericia (Balseiro, Osuna y Javier, 2017).

Son 11 los fármacos que los participantes prescriben y que no se encuentran en el acuerdo emitido por el DOF el día 8 de marzo del 2017, el cual menciona precisamente los fármacos que 


\section{Revista Iberoamericana de las Ciencias de la Salud}

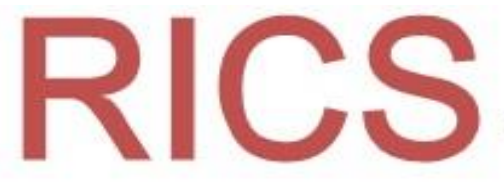

podrán prescribir, los cuales son metoprolol, propranolol, hidróxido de aluminio, omeprazol, cefalexina, isoconazol, clioquinol, nifedipino, lidocaína, ketorolaco y ciprofloxacino; por lo que sería conveniente ampliar el número de fármacos que puedan prescribir los licenciados en enfermería.

La Red Mexicana de Enfermería en la Prescripción de Medicamentos, mencionada por Pérez (2013), no ha tenido ningún tipo de impacto en los profesionales de enfermería de la Costa de Oaxaca; ni siquiera dispone de una página oficial en Internet. Teniendo en cuenta la falta de capacitación en farmacología, ya que más de 90 \% de los participantes mencionó no haber recibido algún curso de actualización en farmacología, cifra similar a la encontrada por Céspedes et al. (2010), quienes refieren que $89.6 \%$ de los encuestados perciben como una necesidad la formación en farmacología, urge que la Secretaría de Salud, junto a la Comisión Permanente de Enfermería, implementen la educación continua sobre la prescripción enfermera para mejorar las atribuciones que el Estado mexicano ha delegado al profesional de enfermería en búsqueda de un mayor empoderamiento del gremio que conforma.

Por lo anterior, se debe considerar que, para prescribir medicamentos, los licenciados en enfermería tendrán que contar con una capacitación continua y actualización en materia de farmacología que permita realizar las actividades profesionales de forma segura y sin riesgos para los pacientes (Balseiro et al., 2017).

Cabe recalcar que Nursing Now, la campaña que está captando la atención de los profesionales de enfermería de todo el mundo, en su cuarto objetivo a alcanzar en el 2020 propone "más enfermeras en posiciones de liderazgo y más oportunidades de desarrollo en todos los niveles". Es triste decirlo, pero no existe en México un profesional de enfermería que asuma la posición de liderazgo en la prescripción enfermera; se necesita apoyar al profesional de enfermería para que lidere, aprenda y utilice todo su potencial en la prescripción (Nursing Now, 2019; Secretaría de Salud, 2018). Esta campaña debe repercutir en los profesionales del área de la Costa de Oaxaca, quienes perciben a la prescripción como una función del gremio, pues todos los aquí encuestados mencionaron que el profesional de enfermería debe prescribir; en contraste con Céspedes et al. (2010), quienes refieren que $62.9 \%$ de sus encuestados no reconoce la prescripción como parte del quehacer de su profesión.

\section{(c) $\mathrm{BP}$ (⿻)}

Esta obra está bajo licencia internacional Creative commons

Reconocimiento-Nocomercial- 


\section{Revista Iberoamericana de las Ciencias de la Salud}

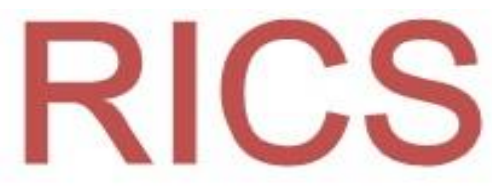

Se plantea mejorar el estudio, obtener una muestra representativa que permita establecer relaciones entre variables a través de un instrumento validado. Asimismo, incluir a licenciados de enfermería que laboren en unidades médicas rurales pertenecientes al IMSS-Bienestar. La presente investigación es única en su tipo en Oaxaca y pretende servir como marco de referencia para implementar políticas de salud en la región Costa. Se observa como debilidad la falta de presupuesto para acceder a los centros de salud de los participantes, la falta de vinculación con otros investigadores, así como la inexistencia de un instrumento validado en México.

\section{Conclusiones}

A partir de los resultados obtenidos en este estudio, se plantea que los licenciados en enfermería que laboran en el primer nivel de atención en centros de salud de la Secretaría de Salud de la región Costa del estado de Oaxaca desconocen el artículo 28 Bis de la Ley General de Salud, y a pesar de ello, prescriben y asumen dicha práctica como una función del gremio. Se establece la imperante necesidad de establecer la educación continua sobre prescripción enfermera, así como guías operativas, manuales, etc.; implementar las bases para la operacionalización organizada; y seguir reformando en cuanto al tema y de acuerdo con las necesidades de la profesión y de la población.

\section{Agradecimientos}

A la Licenciatura en Enfermería de la Universidad de la Costa por las facilidades otorgadas, a los licenciados en enfermería que gustosamente participaron en la recolección de datos y a los profesionales de enfermería que se mantienen mejorando la práctica en beneficio de la salud de las personas.

\section{Referencias}

\section{(C) 9 (क)}


Balseiro, A. L., Osuna, E. y Javier, C. D. (2017). La prescripción de los medicamentos por los licenciados en enfermería: implicaciones de responsabilidad legal. Revista CONAMED, $22(3), 1-9$.

Bellaguarda, M. L., Nelson, S., Padilha, I. M. y Caravaca, A. J. (2015). Autoridad prescriptiva y enfermería: un análisis comparativo de Brasil y Canadá. Revista Latino-Americana de Enfermagem, 23(6), 1065-1073.

Cerezo, M. A., Espinoza, O. E., Techalotzi, A. A. y Tlalpan, R. M. (2016). Elaboración y diseño de la receta de Enfermería. Revista Iberoamericana de las Ciencias de la Salud, 5(10).

Céspedes, L. D., Bedoya, L. C. y Palacios, G. M. (2010). Características de la prescripción por Enfermería en la ciudad de Cali, Colombia. Investigación y Educación en Enfermería, 28(2), 163-170.

Consejo General de Enfermería. (s. f). Marco referencial para la prescripción enfermera. Documento de base. Recuperado de http://www.indexf.com/las casas/documentos/lc0151.pdf

Dandicourt, T. C., Martínez, M. G. y Velásquez, A. A. (2011). Prescripción enfermera, función avanzada para el profesional de enfermería. Revista Horizonte de Enfermería, 22(2), 29-35.

Diario Oficial de la Federación [DOF]. (2012). Decreto por el que se adiciona un artículo 28 Bis a la Ley General de Salud. Diario Oficial de la Federación. Recuperado de http://dof.gob.mx/nota_de talle.php?codigo=523639 9\&fecha=05/03/2012.

Diario Oficial de la Federación [DOF]. (2017). Acuerdo por el que se emiten los lineamientos que contienen el procedimiento y los criterios a los que deberán sujetarse los licenciados en enfermería, así como los pasantes en servicio social de las carreras referidas en los numerales 1 a 5, del artículo 28 Bis, de la Ley General de Salud, para la prescripción de medicamentos. Diario Oficial de la Federación. Recuperado de http://dof.gob.mx/nota_detalle.php?codigo=5475523\& fecha=08/03/2017\&print=true.

Dombrowski, G. J., Pontes, A. J. y Assis, W. A. (2013). Acción de enfermeros en la prescripción de anticonceptivos hormonales en la red de atención primaria de salud. Revista Brasileña de Enfermagem, 66(6), 827-832.

Fernández, M. L. (2016). Prescripción enfermera: situación actual, problemas y alternativas. Fundación Alternativas. Recuperado de http://www.fundacionalternativas.

\section{(c) $\mathrm{GP}$ SP}




\section{Revista Iberoamericana de las Ciencias de la Salud}

org/public/storage/laboratorio_documentos_archivos/3e9d831e3751f125aa4d76e7a9d490 fb.pdf.

Núñez, J. C. (30 de mayo de 2014). Inicia primera reunión nacional de enfermería en la UACH. OMNIA. Recuperado de http://www.omnia.com.mx/noticias/inicia-primera-reunionnacional-de-enfermeria-en-la-uach/.

Nursing Now. (2019). Nuestros objetivos para el 2020. Nursing Now. Recuperado de https://www.nursingnow.org/our-aims/.

Pacheco, R. J. (07 de febrero de 2012). Enfermeros titulados podrán extender recetas médicas. Excelsior. Recuperado de http://www.excelsior.com.mx/2012/02/07/ nacional/808566.

Pérez, H. G. (2013). Prescripción por profesionales de enfermería en México. Revista de Enfermería del Instituto Mexicano del Seguro Social, 21(2), 61-62.

Rodríguez, G. S., de la Fuente, R. N., Casado, M. I. y Ayuso, F. M. (2015). La prescripción enfermera en el sistema Nacional de Salud: ¿realidad o ficción? Enfermería Clínica, 25(3), 107-109.

Royal College of Nursing. (2014). Nurse Prescribing in the UK. Royal College of Nursing. Recuperado de https://www2.rcn.org.uk/_data/assets/pdf_file/0004/462370/15.12 _NursePres cribing_in_the_UK_RCN_Factsheet.pdf.

Secretaría de Salud. (2018). Nursing Now México. Recuperado de http://www.cpe. salud.gob.mx/site3/programa/nursing_now_mexico.html.

Vidal, S. A., Torres, A. M. y Carmena, A. I. (2016). Prescripción enfermera. Beneficios de su implantación. Revista Enfermería Docente, 1(106), 42. Recuperado de http://www.revistaenfermeriadocente.es/index.php/ENDO/article/viewFile/158/pdf_108.

\section{(c) 6 SO}

